\title{
Hiponatremia sintomática como forma de presentación clínica de un macroadenoma hipofisiario. Comunicación de tres casos
}

\author{
Jorge Vega ${ }^{1,2,5}$, Helmuth G oecke ${ }^{1,2,5}$, Alejandra Carrasco ${ }^{1,2}$, \\ Daniela Jensen ${ }^{1}$, Cristián Avilés ${ }^{1}$, Francisca Brusco ${ }^{1,3}$, \\ Renato González ${ }^{1,3}$, Mario Santamarina 4 . \\ Hyponatremia associated to pituitary \\ adenomas. Report of three patients
}

Severe hyponatremia occurring as the presenting feature of hypopituitarism secondary to pituitary adenomas is rare. We report three patients with this condition: Two elderly males (74 and 78 year-old) presenting with impaired consciousness and low plasma sodium after an episode of diarrhea and a 56 year-old male presenting with impaired consciousness after an episode of vomiting. All had clinical features of hypopituitarism and pituitary adenomas were found on imaging studies. Two were subjected to a transsphenoidal resection of the adenoma (Rev Méd Chile 2009, 137: 1607-12).

(Key w ords: Hyponatremia; Hypopituitarism; Pituitary neoplasms)

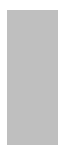

Recibido el 3 de junio, 2009. Aceptado el 27 de octubre, 2009.

${ }^{1}$ Servicio de Medicina, ${ }^{2}$ Secciones de Nefrología, ${ }^{3}$ Endocrinología y ${ }^{4}$ Servicio de Radiología, Hospital Naval A. Nef. Viña del Mar, Chile. ${ }^{5}$ Departamento de Medicina, Escuela de Medicina, Universidad de Valparaíso. Valparaíso, Chile.

L a hiponatremia (HNA) como forma de presentación de un hipopituitarismo (HPT) ha sido comunicada en diversas situaciones clínicas, pero infrecuentemente como primera manifestación de un tumor hipofisario $(\mathrm{TH})^{1-18}$. El objetivo de esta comunicación fue conocer la frecuencia y características clínicas de esta forma de presentación de los THs.

\section{MATERIAL y MÉTODOS}

Entre los años 1990 y 2008 se diagnosticaron 77 TH's y en $3(3,9 \%)$ la HNA fue su primera manifestación clínica. Se registraron los datos demográficos, síntomas y signos consignados en la admisión, medicamentos, exámenes de ingreso e imágenes. Posteriormente, se registraron diariamente estos parámetros, líquidos administrados, diuresis y drogas utilizadas hasta el alta, como la evolución hasta marzo de 2009.

\section{CASOS CLínICOS}

Caso 1. Hombre, 74 años. No ingería medicamentos. Fue hospitalizado por alteración de concien-
Correspondencia a: Dr. Jorge Vega, 5 Norte 1035, Viña del Mar, Chile. Fono: 56-32-2974237. Fax: 56-32-2970050.

E mail: jvegastieb@gmail.com 
cia después de una diarrea autolimitada. Estaba confuso, bradipsíquico, hipotónico, pálido y no había vello axilar. Existía HNA severa (113 mmol/L) con uremia: $23,5 \mathrm{mg} / \mathrm{dl}$, creatininemia: $0,80 \mathrm{mg} / \mathrm{dl}$, uricemia: 2,2 mg/dl y glicemia $109 \mathrm{mg} / \mathrm{dl}$. No recibió sueros el día de ingreso. Al día siguiente, la concentración de sodio en orina: $136 \mathrm{mmol} / \mathrm{L}$, cloro: $121 \mathrm{mmol} / \mathrm{L}$ y potasio: 32,4 mmol/L Recibió $1 \mathrm{~L}$ de solución salina (SS) $0,9 \%$. Al tercer día la natremia fue $112 \mathrm{mmol} / \mathrm{L}$ Se planteó un síndrome de secreción inapropiada de hormona antidiurética (SIADH). Se administró 2 L de SS al 0,9\%. Al cuarto día la natremia era $113 \mathrm{mmol} / \mathrm{L}$ y el paciente continuaba bradipsíquico. Se apreció atrofia de genitales y ausencia de vello pubiano, planteándose un HPT. Se administró hidrocortisona $100 \mathrm{mg} \mathrm{c} / 8 \mathrm{~h}$. Al día siguiente la natremia fue $122 \mathrm{mmol} / \mathrm{L}$ y la conciencia normal. Administrando cortisol oral, $20 \mathrm{mg}(\mathrm{AM})$ y $10 \mathrm{mg}(\mathrm{PM})$, la natremia se normalizó al sexto día $(137 \mathrm{mmol} / \mathrm{L})$. Al reinterrogarlo, el paciente relató disfunción sexual por años, bochornos y reducción de la visión. La evaluación hormonal mostró hipogonadismo hipogonadotrófico, compromiso somatotropo y niveles de cortisol y hormonas tiroideas bajas para una situación de estrés médico, sugerentes de insuficiencia suprarrenal y tiroidea secundarias
(Tabla 1). Un scanner mostró un macroadenoma hipofisiario (Figura 1). Se evidenció hemianopsia bitemporal.

Fue sometido a una resección parcial del tumor por vía trans-esfenoidal. Egresó con suplementación de cortisol, tiroxina y testosterona. Posterior a la cirugía no ha presentado nuevos episodios de HNA.

Caso 2. Hombre de 78 años, en tratamiento con nitrendipino, atorvastatina y aspirina. Consultó en el servicio de urgencia (SU) porque desde hacía un mes presentaba astenia, adinamia, anorexia, fatigabilidad y leve cefalea. Había sufrido una diarrea autolimitada hacía una semana. Al examen presentaba bradipsiquia, ginecomastia, ausencia de vello axilar y escaso en pubis. Había HNA marcada (119 mmol/l) en ausencia de azotemia (uremia: $14 \mathrm{mg} / \mathrm{dl}$, creatininemia: $1,0 \mathrm{mg} / \mathrm{dl}$ ), hiperglicemia y signos clínicos de déficit o exceso de volumen extracelular. En orina sodio: 127 $\mathrm{mmol} / \mathrm{L}$, cloro: $135 \mathrm{mmol} / \mathrm{L}$ y potasio: $37,1 \mathrm{mmol} /$ L. Se administró 1,5 L de SS 0,9\%. Al día siguiente, la natremia persistió baja: $127 \mathrm{mmol} / \mathrm{L}$, planteándose un SIADH o HPT. Se evaluó las funciones tiroidea, suprarrenal e hipofisiaria. Al $3^{\text {er }}$ día se prescribió prednisona $30 \mathrm{mg} /$ día. En los 2 días

Tabla 1. D eterminaciones hormonales en 3 pacientes con tumores hipofisiarios que debutaron con hiponatremia sintomática

\begin{tabular}{|c|c|c|c|}
\hline $\begin{array}{l}\text { Determinaciones hormonales } \\
\text { iniciales }\end{array}$ & $\begin{array}{c}\text { Caso } 1 \\
\text { M acroadenoma } \\
\text { hipofisiario }\end{array}$ & $\begin{array}{c}\text { Caso } 2 \\
\text { M acroadenoma } \\
\text { hipofisiario }\end{array}$ & $\begin{array}{c}\text { Caso } 3 \\
\text { M acroadenoma } \\
\text { hipofisiario }\end{array}$ \\
\hline TSH (VN: 0,35 - 4,94) uU.I./ ml & 0,49 & 0,62 & 2,1 \\
\hline T4 (VN: 4,87 - 11,72) ug/dl & 8,31 & ND & 5,2 \\
\hline T4L (VN: 0,70 - 1,48) ng/dl & 0,80 & 0,70 & ND \\
\hline T3 (VN: 58 - 159) ng/dl & 67 & ND & 35 \\
\hline Testosterona total (VN: $3-12) \mathrm{ng} / \mathrm{dl}$ & 0,66 & 0,70 & ND \\
\hline FSH (VN: $1,55-17,9) \mathrm{mUI} / \mathrm{ml}(\mathrm{M})$ & 2,41 & 5,19 & Normal para la edad* \\
\hline $\mathrm{LH}(\mathrm{VN}: 1,31-10,5) \mathrm{mUI} / \mathrm{ml}(\mathrm{M})$ & 0,74 & 1,85 & Normal para la edad* \\
\hline PRL (VN: 3,7 - 17,9) ng/ml & 5,92 & 23,48 & 16,0 \\
\hline Cortisol AM (VN: 4,2 - 38,4) ug/dl & 5,3 & 2,1 & 14,7 \\
\hline Cortisol PM (VN: 2,5 - 12,5) ug/dl & & ND & 7,6 \\
\hline IGF-1 (VN: $100-494) \mathrm{ng} / \mathrm{ml}$ & 9,5 & ND & ND \\
\hline
\end{tabular}

* Valores no disponibles. ND: determinación no efectuada. 


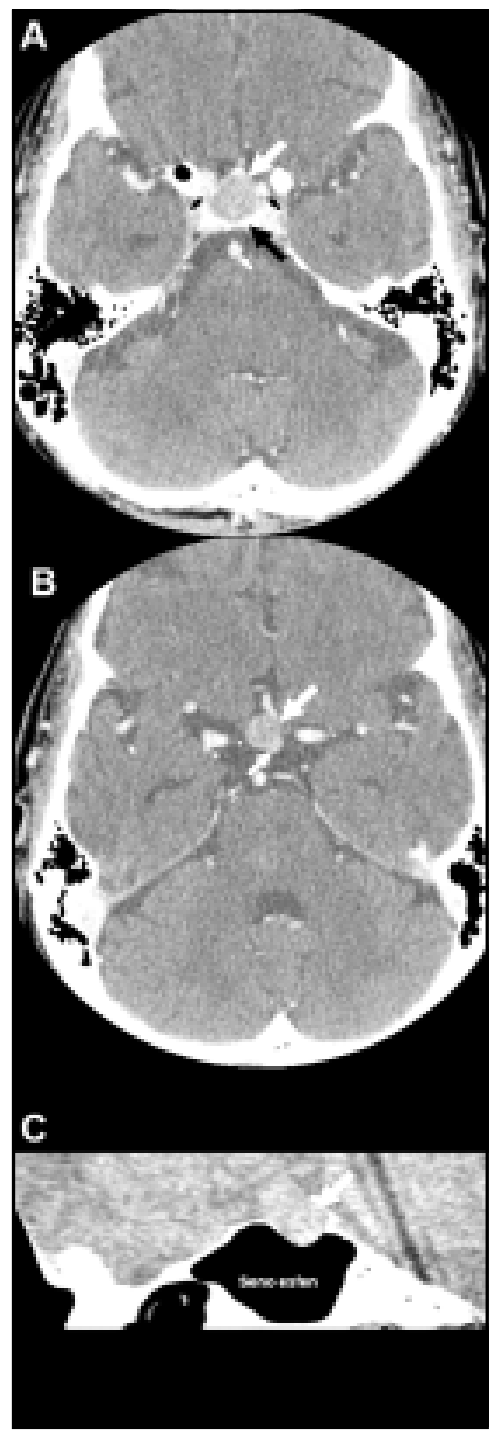

Figura 1. A. Imagen axial de TC con contraste de silla turca. Plano selar. El macroadenoma hipofisiario (flecha blanca grande), produce adelgazamiento de la región del dorso selar (flecha negra grande). Sifones carotídeos (flechas negras pequeñas). Tronco de la arteria basilar (flecha blanca pequeña). No existe invasión evidente de los senos cavernosos. B. Imagen axial de TC con contraste de silla turca. Plano supraselar. Extensión del macroadenoma (flecha blanca grande) hacia la cisterna supraselar. El tallo hipofisiario (flecha blanca pequeña) se encuentra desplazado hacia región posterior. C. Imagen de TC con reconstrucción sagital. Se evidencia que el macroadenoma (flecha blanca), produce importante adelgazamiento del piso de la silla turca. Seno esfenoidal (seno esfen). siguientes, se normalizó la conciencia y la natremia fue $134 \mathrm{mmol} / \mathrm{L}$. Reinterrogado, refirió disfunción eréctil, alejamiento de la afeitada, ginecomastia y caída del vello axilar y pubiano. Las determinaciones hormonales revelaron hipocortisolismo, hipotiroidismo, hipogonadismo e hiperprolactinemia (Tabla 1). La RNM evidenció un TH con compresión del quiasma óptico (Figura 2). Al alta se indicó suplementación de cortisol,

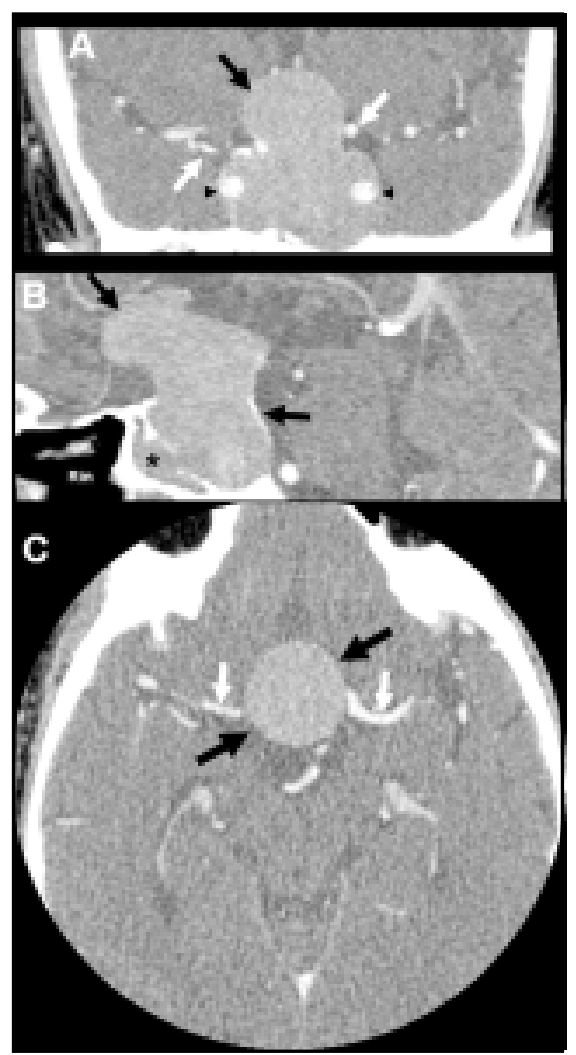

Figura 2. A. Imagen de TC con reconstrucción coronal. Se evidencia voluminosa macroadenoma con realce homogéneo (flecha negra), que ocupa tanto la región intraselar como supraselar. Engloba parcialmente y desplaza hacia la región lateral ambas carótidas intemas, en región del sifón (cabezas de flechas negras). Arterias cerebrales medias (flechas blancas). En región caudal, se evidencia erosión del piso de la silla turca. Hacia anterior, se extiende parcialmente hacia la cisura interhemisférica anterior. B. Imagen de TC con reconstrucción sagital. El macroadenoma (flechas negras) destruye el piso de la silla turca, y se extiende hacia el seno esfenoidal (asterisco). Rinofaringe (Rin). C. Imagen axial de TC. En un plano supraselar, el macroadenoma (flechas negras), ocupa en su totalidad la cistema supraselar. Arterias cerebrales (flechas blancas). 
tiroxina y testosterona, programándose cirugía electiva. Durante el seguimiento la natremia ha permanecido normal.

Caso 3. Hombre, 56 años, con antecedentes de alcoholismo detenido, vit́ligo y glaucoma. Utilizaba timolol. Tres semanas antes del ingreso sufió una bronquitis aguda prescribiéndose cotrimoxazol. Postenormente, apareció dolor abdominal, anorexia, y vómitos que lo llevaron al SU. Estaba confuso y bradipsíquico. Existía HNA marcada (108 mmol/L) en ausencia de hiperglicemia o azotemia (uremia: $30 \mathrm{mg} /$ $\mathrm{dl}$, creatininemia: $1,0 \mathrm{mg} / \mathrm{dl}$ ). En orina la concentración de sodio: $53 \mathrm{mmol} / \mathrm{L}$, cloro: $64,3 \mathrm{mmol} / \mathrm{Ly}$ potasio: 9,1 $\mathrm{mmol} / \mathrm{L}$ Se sospechó HNA por déficit de volumen extracelular y se administró SS 0,9\% con glucosa 5\%. La diuresis fue 5,5 L en 14 horas con balance negativo de $2 \mathrm{~L}$ Al día siguiente, la conciencia había mejorado, pero la natremia persistía baja: $124 \mathrm{mmol} / \mathrm{L}$ Se plantearon SIADH, hipotiroidismo e HPT. El scanner mostró un TH con expansión supraselar, erosión de silla turca y ocupación parcial de la cisterna. El campo visual fue normal, la concentración de T3 claramente disminuida frente a una TSH nomal y la de cortisol baja para una situación de estrés (Tabla 1). En los días siguientes recibió SS al 1,5\% y dieta con sal, normalizando la natremia a los 4 días de su ingreso. La esposa relató que desde hacía un mes el paciente presentaba alteraciones conductuales y labilidad emocional. El paciente refinó disminución de la libido hasta perder completamente el deseo sexual. Egresó a las dos semanas con una natremia normal perdiéndose de los controles. Diecinueve meses después fue intervenido por vía transesfenoidal. En el postoperatorio se demostró hipocortisolismo e hiperprolactinemia, prescribiéndose cortisol. Posteriormente no ha presentado HNA.

\section{Discusión}

Las etiologías de la HNA se clasifican según el estado del volumen extracelular (VEC). Las asociadas a un VEC clínicamente normal comprenden a las secundarias a SIADH, drogas, náuseas persistentes, dolor crónico y deficiencias endocrinas (hipopituitarismo e hipotiroidismo) ${ }^{19}$.

El hipotiroidismo primario rara vez se asocia a HNA y cuando lo hace, generalmente ocurre en sujetos añosos que cumplen criterios de coma mixedematoso ${ }^{20}$. La causa principal es un defecto en la excreción de agua por una alteración en la perfusión renal y caída en la velocidad de filtración glomerular secundaria a los efectos del déficit de la hormona tiroidea en el gasto cardíaco y en la resistencia vascular periférica ${ }^{21}$. Ello origina un estímulo para la secreción de la ADH por activación de barorreceptores ${ }^{22}$. Cuando la HNA acompaña al HPT usualmente ésta es una manifestación de la insuficiencia suprarrenal secundaria más que del hipotiroidismo secundario coexistente.

Aunque los TH's son una causa común de hipopituitarismo, la HNA sintomática rara vez es su motivo de presentación clínica ${ }^{6,7}$. La HNA se ha observado asociada a adenomas, craneofaringiomas, prolactinomas, quistes, schwanomas, meningiomas, aneurismas, carcinoma nasofaríngeo y metástasis de carcinomas $8,12,16-18,23-28$.

La HNA asociada a TH's mayoritariamente se ha descrito en mayores de 70 años. La capacidad secretora de la pituitaria es menor en ellos, especialmente cuando es comprimida por un tumor grande ${ }^{6,14}$. También, la liberación de ADH frente a estímulos fisiológicos y farmacológicos es mayor en individuos añosos ${ }^{29}$. En nuestra serie 2 de los 3 pacientes fueron mayores de 70 años y la causa de la HNA fue un HPT originado en un macroadenoma hipofisiario. En ellos se evidenció un déficit de hormonas pituitarias (Tabla 1) y su sustitución hizo que la HNA se resolviera en pocos días. En el tercer paciente, es probable que un déficit de VEC haya sido causante de la HNA, ya que se normalizó con SS sin sustitución hormonal. No es descartable que haya existido un déficit de glucocorticoides, dada la dificultad en normalizar la natremia con SS hipertónica. No se efectuó test de estimulación con ACTH o hipoglicemia inducida por insulina, por lo que no puede descartarse que haya existido un daño del eje ACTH-adrenal.

Alrededor de 30\% de los TH's son del tipo no secretor $^{26}$, como en los pacientes de esta serie. Usualmente crecen lentamente y comprometen estructuras vecinas. Cuando afectan la función, la hormona de crecimiento (GH) es la primera comprometida. Posteriormente le siguen las gonadotrofinas, ACTH y TSH ${ }^{30}$. La prolactina (PRL) y la hormona antidiurética (ADH) permanecen sin afectarse o están aumentadas. Los primeros síntomas de deficiencia hormonal (gonadotrofinas) son amenorrea y en los hombres, disminución de la energía, reducción de la líbido y potencia sexual por déficit de testosterona ${ }^{18}$. En esta serie, todos 
presentaban disfunción sexual y uno de ellos atrofia de genitales. En una serie de 28 pacientes con HPT e HNA recolectados durante 19 años (6 TH's), el signo clínico más frecuente fue la ausencia 0 escasez de vello axilar y pubiano ${ }^{18}$, como ocurrió en 2 de nuestros enfermos.

La deficiencia de ACTH a menudo es asintomática y sólo se desenmascara en presencia de estrés o drogas ${ }^{18}$. Los 3 pacientes de esta serie presentaron HNA después de cuadros digestivos o infección bronquial. Es probable que en ellos se haya descompensado una deficiencia crónica de glucocorticoides por el estrés originado por estos trastornos o que los síntomas digestivos hayan sido la manifestación inicial de la insuficiencia suprarrenal. Cuando el adenoma se extiende a la región supraselar, pueden aparecer alteraciones visuales, lo que ocurrió sólo en 1 de nuestros 3 pacientes. Esto ha hecho especular a algunos autores que la HNA severa es muy infrecuente como forma de presentación de los tumores no funcionantes porque es una manifestación clínica muy tardía ${ }^{7}$. En ocasiones, los episodios de HNA han sido recurrentes, previo al diagnóstico del $\mathrm{TH}^{15,18}$.

El mecanismo de producción de la HNA en el HPT es la secreción exagerada de hormona antidiurética $(\mathrm{ADH})$, cumpliéndose casi todos los criterios diagnósticos de un SIADH salvo la indemnidad de las funciones tiroideas y supramenal ${ }^{8}$. Esto permite diferenciar a las hiponatremias originadas en SIADH de las causadas por un HPT. La uricemia baja se debe a la expansión de volumen que origina una excreción aumentada de ácido úrico en la orina. En esta serie los 3 pacientes tuvieron sodio urinario inapropiadamente

\section{REFERENCIAS}

1. Putterman C, Alomg Y, Caraco Y, Gross Dj, Ben-chetrit E. Inappropriate secretion of antidiuretic hormone in Sheehan's syndrome: a rare cause of post-partum hiponatremia. Am J Obstet Gynecol 1991; 165: 1330-3.

2. Michilis A, BALERIAUX D, MocKel J. Bilateral carotid aneurysms unmasked by severe hypopituitarism. Postgrad Med 1991; 67: 285-8.

3. DAVIES JS, SCANLON MF. Hypopituitarism after coronary artery bypass grafting. BMJ 1998; 316: 682-5.

4. OKuno S, Inaba M, Nishizana Y, Miki T, Inoue Y, MoriI H. A case of hyponatremia in panhypopituitarism elevado para la severidad de la HNA y en 2 de ellos el aporte de SS no comigió el trastomo. El cortisol endógeno ejerce un efecto inhibidor tónico sobre la secreción de $\mathrm{ADH}^{18}$. En la deficiencia de glucocorticoides hay una liberación de $\mathrm{ADH}$ no supresible a pesar de la hipo-osmolalidad existente ${ }^{29}$.

En estudios experimentales, la deficiencia de cortisol se relaciona con aumento de la liberación de ADH. Los antagonistas de ADH revierten casi completamente la incapacidad de excretar agua y el reemplazo de los glucocorticoides, rápidamente incrementa su excreción. La ADH parece cosecretarse con el factor liberador de corticotropina (CRF) por las células del núcleo paraventricular. Así, los glucocorticoides producen retroalimentación negativa tanto en la liberación de CRF como de $\mathrm{ADH}^{31-33}$. El mARN y proteínas de los canales de agua (aquaporina-2) están sobre-expresados en ratas deficientes en corticoides. El aporte de hidrocortisona corrige el defecto en la excreción de agua y normaliza los niveles de ADH y la expresión renal de mARN de aquaporina $2^{34}$.

La mayoría de los pacientes con HNA asociada a HPT tienen una excreción aumentada de aquaporina-2, que es reflejo de la acción de ADH en el túbulo colector y ella cae al administrar hidrocortisona ${ }^{18,34}$. En 2 de nuestros pacientes, los glucocorticoides incrementaron la diuresis $y$ normalizaron la natremia en 5-6 días.

En suma, el HPT debe ser considerado en las HNA de etiología no explicada. Los hallazgos clínicos y bioquímicos son indistinguibles de un SIADH, sin embargo su tratamiento es diferente. Los corticoides la revierten en pocos días.

caused by the primary empty sella. Endocrinol Jpn 1987; 34: 299-307.

5. Lam KSL, Kung AWC, Young RTT. Post-irradiation hypopituitarism presenting as severe hyponatremia. Am J Med 1992; 92: 219-21.

6. Nishizawa S, Yokoyama T, Yokota N, Ohta S. Preoperative hyponatremia as a clinical characteristic in elderly patients with large pituitary tumor. Neurol Med Chir (Tokyo) 2000; 40: 249-55.

7. LiN SH, Hung YH, LN YF. Severe hyponatremia as the presenting feature of clinically non-functional pituitary adenoma with hypopituitarism. Clin $\mathrm{Ne}-$ phrol 2002; 57: 85-8. 
8. Kanda M, Omori Y, Shinoda S, Yamauchi T, Tamemoto $\mathrm{H}$, KaWAKAMI M ET AL. SIADH closely associated with non-functioning pituitary adenoma. Endocr J 2004; 51: 435-8.

9. Pitale SU, Lee JM, Origitano T, Emanuele NV. An unusual pituitary mass presenting with panhypopituitarism and hyponatremia. Postgrad Med J 2001; 77: 530-1, 539-40.

10. Tripathi M, Sanjeev CC, Roy AK. Chasing hyponatremia: unusual presentation. Neurol India 2001; 49: 197-9.

11. Haraguchi K, Morimoto S, Tanooka A, Inoue $M$, Yoshida Y. Craniopharyngioma presenting a symptom of pituitary apoplexy and hyponatremia: a case report. No Shinkei Geka 2000; 28: 1111-5.

12. Hung SC, WEN YK, NG YY, YANG WC. Inappropriate antidiuresis associated with pituitary adenoma-mechanisms not involving inappropriate secretion of vasopressin. Clin Nephrol 2000; 54: 157-60.

13. Pontuch P, Vestenická V, Sediák T, Porubec V, Kinová $\mathrm{S}$, JEzoví $\mathrm{D}$. The syndrome of inappropriate secretion of antidiuretic hormone in hypophyseal tumours. Vnitr Lek 1998; 44: 104-7.

14. Gonzales-Portilo G, Tomta T. The syndrome of inappropriate secretion of antidiuretic hormone: an unusual presentation for childhood craniopharyngioma: report of three cases. Neurosurgery 1998; 42: 917-21.

15. Yamashino S, Fuwa I, Seto H, Ushio Y. Initial and postoperative hyponatremia associated with pituitary adenoma: a case report. Acta Neurochir (Wien) 1997; 139: 987-90.

16. Bernreuther C, Futsch J, Lûdecke DK, Hagel C. A 61year-old man with hyponatremia. Brain Pathol 2008; 18: 283-7.

17. Saito T, Watanabe $Y$, Yuzana $M$, Saito T, Tamemoto $H$, SuzUKI H ET AL. SIADH is only an atypical clinical feature in a patient with prolactinoma. Intern Med 2007; 46: 653-6.

18. Diederich S, Franzen NF, Bahr V, Oelkers W. Severe hyponatremia due to hypopituitarism with adrenal insufficiency: report on 28 cases. Eur J Endocrinol 2003; 148: 609-17.

19. Verbalis JG, Goldsmith SR, Greenberg A, Schrier RW, STERNS RH. Hyponatremia treatment guidelines 2007: Expert panel recommendations. Am J Med 2007; 120 (11A); S1-S21.

20. Chinitz A, Turner FL The association of primary hypothyroidism and inappropriate secretion of the antidiuretic hormone. Arch Intern Med 1965; 116: 871-4.
21. Derubertis FR, Michelin MF, Bloom ME, Mintz DH, FELD JB, Davis BB. Impaired water excretion in myxedema. Am J Med 1971; 51: 41-53.

22. Chen YC, CAdnapaphornchai MA, Yang J et aL. Nonosmotic release of vasopressin and renal aquaporins in impaired urinary dilution in hypothyroidism. Am J Physiol 2005; 289: F672-F678.

23. Beigel R, Shiff E, Luckman J, Dessau H. Hyponatremia as a presenting sign of a pituitary intrasellar cyst. Isr Med Assoc J 2005; 7: 126-7.

24. Chien WY, Wang PW, Huang HS, Huang MJ. Aneurysm of the internal carotid artery simulating pituitary tumour with panhypopituitarism- a case report. Changgeng Yi Xue Za Zhi 1989; 12: 161-6.

25. IzUmi $Y$, Sakagushi $K$, Udaka F, Tsujimura T, Kameyama $M$. A patient with meningeal carcinomatosis accompanied by a small pituitary metastatic lesion from gastric cancer who developed cerebral salt wasting syndrome. Nippon Ronen Igakkai Zasshi 1999; 36: 657-62.

26. KoKatsu T, Tsukuda M. Hypopituitarism and hyponatremia in a case with nasopharyngeal carcinoma. ORLJ Otorhinolaryngol Relat Spec 1994; 56: 347-51.

27. Lu H, Yamaki T, OKa S, Koyanagi I, Houkin K. Metastatic renal cell carcinoma mimicking pituitary adenoma: case report. Neurol Med Chir (Tokyo) 2005; 45: 418-22.

28. Kanamata T, Harashima S, Kubo O, Hori T. Intrasellar remote metastasis from adenoid cystic carcinoma of parotid gland: case report. Endocr J 2006; 53: 659-63.

29. Yatagai T, Kusaka I, Nakamura T, Nagasaka S, Honda $\mathrm{K}$, ISHIBASHI S ET AL. Close association of severe hyponatremia with exaggerated release of arginine vasopressin in elderly subjects with secondary adrenal insufficiency. Eur J Endocrinol 2003; 148: 221-6.

30. PETER JS. Clinically non-functioning pituitary adenomas. Endocrinol Metab Clin North Am 1993; 22: 163-75.

31. Pham PC, Pham PA, Pham PT. Sodium and water disturbances in patients with Sheehan's syndrome. Am J Kidney Dis 2001; 38: E14.

32. ISHIKAWA SE, SCRIER RW. Effect of arginine vasopressin antagonist on water excretion in glucocorticoid and mineralocorticoid -deficient rats. Kidney Int 1982; 22: 587-93.

33. RAFF H. Glucocorticoid inhibition of neurohypophyseal vasopressin secretion. Am J Physiol 1987; 252: R635-R644.

34. Saito T, Ishikana Se, Ando F, Okada N, Nakamura T, KusAKa I ET AL. Exaggerated urinary excretion of aquaporin-2 in the pathological state of impaired water excretion dependent upon arginine vasopressin. J Clin Endocrinol Metab 1998; 83: 4034-40. 